\begin{tabular}{|c|l|}
\hline Title & $\begin{array}{l}\text { Momentum operators with gauge potentials, local quantization of magnetic flux, and representation of canonical } \\
\text { commutation relations }\end{array}$ \\
\hline Author(s) & A rai, A sao \\
\hline Citation & $\begin{array}{l}\text { Journal of Mathematical Physics, 33(10), 3374-3378 } \\
\text { https://doi.org/10.1063/1.529938 }\end{array}$ \\
\hline Issue Date & 1992-10 \\
\hline Doc URL & http://hdl.handle.net/2115/13677 \\
\hline Rights & Copyright $\odot 1992$ A merican Institute of Physics \\
\hline Type & article \\
\hline File Information & jmp33-10.pdf \\
\hline
\end{tabular}

Instructions for use 


\title{
Momentum operators with gauge potentials, local quantization of magnetic flux, and representation of canonical commutation relations
}

\author{
Asao Arai \\ Department of Mathematics, Hokkaido University, Sapporo 060, Japan
}

(Received 4 February 1992; accepted for publication 20 May 1992)

\begin{abstract}
Commutation properties of two-dimensional momentum operators with gauge potentials are investigated. A notion of local quantization of magnetic flux is introduced to characterize physically the strong commutativity of the momentum operators. In terms of the notion, a necessary and sufficient condition is given for the position and the momentum operators to be equivalent to the Schrödinger representation of the canonical commutation relations.
\end{abstract}

\section{INTRODUCTION}

It was shown in Ref. 1 that a representation of the canonical commutation relations (CCRs) inequivalent to the Schrödinger representation occurs in connection with a planar quantum system with a perpendicular magnetic ficld concentrated on the origin. The inequivalent representation comes from the noncommutativity (in the strong sense) of the momentum operators with a singular gauge potential of vortex type; "Nelson's phenomenon"2 occurs for the momentum operators. It is interesting to find more gauge potentials that give rise to such a phenomenon and to investigate general mathematical structures underlying it. This is the basic motivation of the present work.

We mention that, in the case of one degree of freedom, Schmüdgen ${ }^{3,4}$ has given a detailed analysis on representations of the CCR inequivalent to the Schrödinger representation, together with some examples, ${ }^{4}$ one of which is in the spirit of Nelson's example. ${ }^{2}$ For works related to representations of the CCRs, see the references given in Refs. 3 and 4.

The outline of the present paper is as follows. In Sec. II, we start our analysis with a general two-dimensional quantum system with a gauge potential that may be singular at some isolated points in the two-dimensional space $\mathbf{R}^{2}$. The main point is to investigate the commutation relations (in the strong sense) of the momentum operators with the gauge potential. We introduce a notion of local quantization of magnetic flux. We show that, under some conditions, the momentum operators strongly commute if and only if the magnetic flux is locally quantized. This gives a physical characterization of the strong commutativity of the momentum operators. Also, for a class of gauge potentials, a necessary and sufficient condition is given for the magnetic flux to be locally quantized. In Sec. III, we define classes of gauge potentials, which contain the example used in Ref. 1, and discuss some examples. In Sec. IV, we apply the preceding results to representation of the CCRs to prove that, under some conditions, the position and the momentum operators fulfills the Weyl relations if and only if the magnetic flux is locally quantized. This generalizes the main result in Ref. 1.

\section{MOMENTUM OPERATORS WITH GAUGE POTENTIALS AND LOCAL QUANTIZATION OF MAGNETIC FLUX}

We consider a quantum system of a spinless charged particle with charge $q \in \mathbf{R} \backslash\{0\}$ moving in the plane $\mathbf{R}^{2}$ under the influence of a perpendicular magnetic field. We denote by $\mathbf{A}(\mathbf{r})=\left(A_{1}(\mathbf{r}), A_{2}(\mathbf{r})\right)\left[\mathbf{r}=(x, y) \in \mathbf{R}^{2}\right]$ a gauge potential of the magnetic field. We consider the situation where $A_{j}(j=1,2)$ may be singular at the points $a_{n}$ $=\left(a_{n 1}, a_{n 2}\right) \in \mathbf{R}^{2}, n=1, \ldots, N$. Let

$$
\mathbf{\Omega}=\mathbf{R}^{2} \backslash\left\{\mathbf{a}_{1}, \ldots, \mathbf{a}_{N}\right\} \text {. }
$$

Throughout this section, we assume the following.

Assumption $A$ : Each $A_{j}(j=1,2)$ is in $C(\Omega)$ (the space of continuous functions on $\Omega$ ).

Remark: Under Assumption A, it is natural to treat the magnetic field $B$ as a distribution. Hence, denoting by $D_{x}$ and $D_{y}$ the distributional partial differential operators in $x$ and $y$, respectively, we may define $B$ by

$$
B(\mathbf{r})=D_{x} A_{2}(\mathbf{r})-D_{y} A_{1}(\mathbf{r}),
$$

provided that $A_{1}$ and $A_{2}$ are distributions on $\mathbf{R}^{2}$.

The purpose of this section is to investigate commutation relations of the momentum operators $P_{1}$ and $P_{2}$ defined by

$$
\begin{aligned}
& P_{1}=p_{1}-q A_{1}, \\
& P_{2}=p_{2}-q A_{2},
\end{aligned}
$$

with

$$
p_{1}=-i D_{x}, \quad p_{2}=-i D_{y},
$$


where we use a system of units in which the light speed $c$ and the Planck constant $h$ are equal to one. To state the main result, we need some definitions. For $x, y, s, t \in \mathbf{R}$, we define two curves $C_{ \pm}(x, y ; s, t)$ from $(x, y) \in \mathbf{R}^{2}$ to $(x+s, y$ $+t) \in \mathbf{R}^{2}$ by

$$
\begin{aligned}
C_{-}(x, y ; s, t) & =\{(x+\theta s, y) \mid 0 \leqslant \theta \leqslant 1\} \\
\cup\{(x+s, y+\theta t) \mid 0 \leqslant \theta \leqslant 1\}, & \\
C_{+}(x, y ; s, t) & =\{(x, y+\theta t) \mid 0 \leqslant \theta \leqslant 1\} \\
& \cup\{(x+\theta s, y+t) \mid 0 \leqslant \theta \leqslant 1\} .
\end{aligned}
$$

Let

$$
C(x, y ; s, t)=C_{-}(x, y ; s, t)-C_{+}(x, y ; s, t),
$$

which is the rectangular closed curve:

$$
(x, y) \rightarrow(x+s, y) \rightarrow(x+s, y+t) \rightarrow(x, y+t) \rightarrow(x, y) .
$$

Let

$$
\mathbf{S}_{j}^{(t)}=\mathbf{R} \backslash\left\{a_{n j} a_{n j}-t\right\}_{n=1}^{N}, \quad j=1,2, \quad t \in \mathbf{R},
$$

and

$$
\Omega_{s, t}=\mathbf{S}_{1}^{(s)} \times \mathbf{S}_{2}^{(t)}, \quad s, t \in \mathbf{R} .
$$

For each $s, t \in \mathbf{R}$, we define a function $\Phi_{s, t}^{\mathrm{A}}$ on $\Omega_{s, t}$ by

$$
\Phi_{s, t}^{A}(x, y)=\int_{C(x, y ; s, t)} \mathbf{A}\left(\mathbf{r}^{\prime}\right) \cdot d \mathbf{r}^{\prime}
$$

which physically means the flux of the magnetic field $B$ going through the interior of $C(x, y ; s, t)$. Since the Lebesgue measure of the set $\mathbf{R}^{2} \backslash \Omega_{s, t}$ is zero, the function $\Phi_{s, t}^{\mathbf{A}}(x, y)$ is defined for a.e. (almost everywhere) $(x, y) \in \mathbf{R}^{2}$ and real valued. Hence it defines a unique, self-adjoint, multiplication operator on $L^{2}\left(\mathbf{R}^{2}\right)$. We denote it by the same symbol $\Phi_{s, t}^{\mathbf{A}}$

We shall denote by $D(T)$ the domain of the operator $T$. The main result in this section is the following.

Theorem 2.1: Suppose that each $P_{j}(j=1,2)$ is essentially self-adjoint on $D\left(p_{j}\right) \cap D\left(A_{j}\right)$ and denote the closure of $P_{j}$ by $\bar{P}_{j}$. Then, for all $s, t \in \mathbf{R}$,

$$
e^{i s \bar{P}_{1}} e^{i t \bar{P}_{2}}=\exp \left(-i q \Phi_{s, t}^{\mathbf{A}}\right) e^{i t \bar{P}_{2}} e^{i s \bar{P}_{1}} .
$$

Proof: By the assumption, we can apply the Trotter product formula (e.g., Theorem VIII.31 in Ref. 5) to $P_{1}$ and $P_{2}$ to obtain

$$
\begin{gathered}
\left(\Phi, e^{i t \bar{P}_{J}} \Psi\right)=\lim _{n \rightarrow \infty}\left(\Phi,\left(e^{i t p_{j} / n} e^{-i t A_{j} / n}\right)^{n} \Psi\right), \\
\Phi, \Psi \in L^{2}\left(\mathbf{R}^{2}\right), \quad t \in \mathbf{R},
\end{gathered}
$$

where $(\cdot, \cdot)$ denotes the inner product of $L^{2}\left(\mathbf{R}^{2}\right)$. Using the fact that

$$
\left(e^{i s p_{1} \Psi}\right)(x, y)=\Psi(x+s, y), \quad \text { a.e. }(x, y) \in \mathbf{R}^{2}, \quad s \in R,
$$

we can show that

$$
\begin{aligned}
& \left(e^{i s p_{1} / n} e^{-i s q A_{1} / n}\right)^{n} \Psi(x, y) \\
& \quad=\exp \left[-i q \sum_{k=1}^{n} \frac{s}{n} A_{1}\left(x+\frac{k s}{n}, y\right)\right] \Psi(x+s, y) .
\end{aligned}
$$

Hence we have for a.e. $(x, y) \in \mathbf{R}^{2}$

$$
\begin{aligned}
& \lim _{n \rightarrow \infty}\left(e^{i s p_{1} / n} e^{-i s q A_{1} / n}\right)^{n} \Psi(x, y) \\
& \quad=\exp \left(-i q \int_{0}^{s} A_{1}\left(x+x^{\prime}, y\right) d x^{\prime}\right) \Psi(x+s, y),
\end{aligned}
$$

and

$$
\left|\left(e^{i s p_{1} / n} e^{-i s q A_{1} / n}\right)^{n} \Psi(x, y)\right| \leqslant|\Psi(x+s, y)| .
$$

These facts allow us to apply the Lebesgue dominated convergence theorem to obtain

$$
\begin{aligned}
\left(\Phi, e^{i s \bar{P}_{1}} \Psi\right)= & \int_{\mathbf{R}^{2}} \Phi(x, y)^{*} \exp \left(-i q \int_{0}^{s} A_{1}\left(x+x^{\prime}, y\right) d x^{\prime}\right) \\
& \times \Psi(x+s, y) d x d y,
\end{aligned}
$$

which implies that

$$
\begin{aligned}
e^{i s \bar{P}_{1}} \Psi(x, y)= & \exp \left(-i q \int_{0}^{s} A_{1}\left(x+x^{\prime}, y\right) d x^{\prime}\right) \\
& \times\left(e^{\left.i s p_{1} \Psi\right)(x, y) .}\right.
\end{aligned}
$$

Similarly we can prove that

$$
\begin{aligned}
e^{i t \vec{P}_{2}} \Psi(x, y)= & \exp \left(-i q \int_{0}^{t} A_{2}\left(x, y+y^{\prime}\right) d y^{\prime}\right) \\
& \times\left(e^{i t p_{2} \Psi}\right)(x, y) .
\end{aligned}
$$

Using these formulas, we obtain

$$
e^{i s \bar{P}_{1}} e^{i t \overline{P_{2}}}=\exp \left(-i q \int_{C_{-}(x, y ; s, t)} \mathbf{A}\left(\mathbf{r}^{\prime}\right) \cdot d \mathbf{r}^{\prime}\right) e^{i s p_{1}} e^{i t p_{2}}
$$

and 


$$
e^{i t \bar{P}_{2}} e^{i s \bar{P}_{1}}=\exp \left(-i q \int_{C_{+}(x, y, s, t)} \mathbf{A}\left(\mathbf{r}^{\prime}\right) \cdot d \mathbf{r}^{\prime}\right) e^{i t P_{2}} e^{i s p_{1}}
$$

Since $e^{i s p_{1}}$ and $e^{i t p_{2}}$ commute, (2.1) follows.

Remark: If $A_{j}$ is relatively bounded with respect to $p_{j}$ with a relative bound less than one, then it follows from the Kato-Rellich theorem (e.g., Sec. X.2 in Ref. 6) that $P_{j}$ is essentially self-adjoint on $C_{0}^{\infty}\left(\mathbf{R}^{2}\right)$. Hence, in this case, the assumption of Theorem 2.1 is satisfied. More singular gauge potentials that satisfy the assumption of Theorem 2.1 will be considered in Sec. III.

We next discuss implications of Theorem 2.1. For this purpose, we first recall a proper definition of commutativity of self-adjoint operators (e.g., Sec. VIII.5 in Ref. 5): Two self-adjoint operators $A$ and $B$ are said to strongly commute if

$$
e^{i s A} e^{i t B}=e^{i t B} e^{i s A}
$$

for all $s, t \in \mathbf{R}$. This notion of commutativity and Theorem 2.1 lead us to the following definition.

Definition 2.2: Let $\mathbf{Z}$ be the set of integers. We say that the magnetic flux associated with a vector potential A is locally quantized if $\Phi_{s, t}^{\mathbf{A}}$ is a $2 \pi \mathbf{Z} / q$-valued function for all $s, t \in \mathbf{R}$.

Remarks: (i) The local quantization property of magnetic flux is gauge invariant.

(ii) The standard notion of quantization of magnetic flux is a global one, i.e., it is concerned with a quantization of the total magnetic flux defined by

$$
\Phi^{\mathbf{A}}=\lim _{x, y \rightarrow-\infty, s, t \rightarrow \infty} \Phi_{s, t}^{\mathbf{A}}(x, y),
$$

provided that the limit exists. If the magnetic flux associated with $\mathbf{A}$ is locally quantized, then $\Phi^{\mathbf{A}}$ is quantized. However, the converse is not true.

The following fact immediately follows from Theorem 2.1 .

Corollary 2.3: Under the assumption of Theorem 2.1, $\overrightarrow{P_{1}}$ and $\bar{P}_{2}$ strongly commute if and only if the magnetic flux associated with $\mathbf{A}$ is locally quantized.

Since the notion of local quantization of magnetic flux is a physical one, Corollary 2.3 gives a physical characterization of the strong commutativity of the momentum operators.

In view of Corollary 2.3, it is important to know for what gauge potentials the magnetic fluxes associated with them are locally quantized. We next consider this problem. Let $D(x, y ; s, t)$ be the interior of $C(x, y ; s, t)$ and

$$
\epsilon(s)= \begin{cases}1, & s \geqslant 0 \\ -1, & s<0\end{cases}
$$

Take a constant $\delta>0$ to be sufficiently small such that for all $j, k=1, \ldots, N$ with $j \neq k$,

$$
\left\{\mathbf{r}|| \mathbf{r}-\mathbf{a}_{j} \mid \leqslant \delta\right\} \cap\left\{\mathbf{r}|| \mathbf{r}-\mathbf{a}_{k} \mid \leqslant \delta\right\}=\varnothing,
$$

and set

$$
\gamma_{n}(\mathbf{A})=\int_{\left|\mathbf{r}-\mathbf{a}_{n}\right|=\delta} \mathbf{A}(\mathbf{r}) \cdot d \mathbf{r},
$$

where the orientation of the contour $\left|\mathbf{r}-\mathbf{a}_{n}\right|=\delta$ is taken to be anticlockwise.

Lemma 2.4: Let $\mathbf{A} \in C^{1}\left(\Omega ; \mathbf{R}^{2}\right)$ (the space of $\mathbf{R}^{2}$. valued, continuously differentiable functions on $\Omega$ ) and suppose that

$$
B(\mathbf{r})=0, \quad \mathbf{r} \in \Omega .
$$

Then $\gamma_{n}(\mathbf{A})$ is independent of $\delta$ and the following formula holds:

$$
\Phi_{s, t}^{\mathbf{A}}(x, y)=\epsilon(s) \epsilon(t) \sum_{\mathbf{a}_{n} \in D(x, y ; s, t)} \gamma_{n}(\mathbf{A}), \quad(x, y) \in \Omega_{s, t} .
$$

Proof: This follows from an application of Green's theorem.

The following theorem gives a necessary and sufficient condition for the magnetic flux associated with a gauge potential to be locally quantized.

Theorem 2.5: Let $A \in C^{1}\left(\Omega ; \mathbf{R}^{2}\right)$. Then the magnetic flux associated with $A$ is locally quantized if and only if (2.2) is satisfied and $\gamma_{n}(\mathbf{A})$ is an integer multiple of $2 \pi / q$ for all $n=1, \ldots, N$.

Proof: Suppose that the magnetic flux associated with $A$ is locally quantized. For any $(x, y) \in \Omega$, there exists a constant $c=c(x, y)>0$ such that for all $s, t \in[-c, c]$, and $n=1, \ldots, N, \mathbf{a}_{n} \notin C(x, y ; s, t) \cup D(x, y ; s, t)$. By Grcen's theorem, we have

$$
\Phi_{s, t}^{\mathbf{A}}(x, y)=\iint_{D(x, y ; s, t)} B\left(\mathbf{r}^{\prime}\right) d \mathbf{r}^{\prime}, \quad|s|,|t| \leqslant c .
$$

It follows from this formula that

$$
\lim _{s, t 10} \frac{\Phi_{s, t}^{\mathbf{A}}(x, y)}{s t}=B(x, y) .
$$

Using (2.4), we can also show that

$$
\left|\Phi_{s, t}^{\mathbf{A}}(x, y)\right| \leqslant d|s||t|, \quad|s|,|t| \leqslant c,
$$

with some constant $d=d(c, x, y)>0$. Under the present assumption, $\Phi_{s, t}^{\mathbf{A}}(x, y)$ is a $2 \pi \mathbf{Z} / q$-valued function as a function of $(s, t)$. Hence (2.6) implies that $\Phi_{s, t}^{\mathbf{A}}(x, y)$ $=0$ for all sufficiently small $|s|,|t|$. This fact and (2.5) give (2.2). Hence, by Lemma 2.4, (2.3) holds. Since $\Phi_{s, t}^{\mathbf{A}}$ is a $2 \pi \mathbf{Z} / q$-valued function for all $s, t \in \mathbf{R}$, it follows that each $\gamma_{n}(\mathbf{A})$ must be an integer multiple of $2 \pi / q$. 
Conversely, if (2.2) is satisfied and $\gamma_{n}(\mathbf{A})$ is an integer multiple of $2 \pi / q$ for all $n=1, \ldots, N$, then, by Lemma $2.4, \Phi_{s, t}^{\Lambda}$ is a $2 \pi \mathrm{Z} / q$-valued function for all $s, t \in \mathbf{R}$.

It is easy to see that, under condition (2.2), $\gamma_{n}(\mathbf{A})$ can be different from zero only if $\mathbf{A}$ is singular at $\mathbf{r}=\mathbf{a}_{n}$. Hence, Theorem 2.5 shows that, for the magnetic flux associated with a vector potential $A \in C^{1}\left(\Omega ; \mathbf{R}^{2}\right)$ to be locally quantized in a non-trivial way, $\mathbf{A}$ must have singularities at some of $\mathbf{a}_{n}$ 's.

Remark: If $B$ is a distribution on $\mathbf{R}^{2}$ and satisfies (2.2), then it follows from a general theorem in distribution theory (e.g., Chap. II, Sec. 4.5 in Ref. 7) that $B$ must be of the form

$$
B(\mathbf{r})=\sum_{n=1}^{N} \sum_{\alpha, \beta=0}^{k} \lambda_{\alpha \beta}^{(n)} D_{x}^{\alpha} D_{y}^{\beta} \delta\left(\mathbf{r}-\mathbf{a}_{n}\right),
$$

where $k$ is a non-negative integer and $\lambda_{\alpha \beta}^{(n)}, \alpha, \beta=1, \ldots, k$, $n=1, \ldots, N$, are real constants. A gauge potential that gives such a magnetic field is discussed in Sec. III.

\section{CLASSES OF SINGULAR GAUGE POTENTIALS}

In this section we show that there exist classes of singular gauge potentials that satisfy the assumption of Theorem 2.1 and discuss some examples. Let

$$
\mathbf{S}_{j}=\mathbf{R} \backslash\left\{a_{n j}\right\}_{n=1}^{N}, \quad j=1,2 .
$$

For an open set $\mathbf{M} \subset \mathbf{R}^{2}$, we denote by $C^{m}(\mathbf{M})$ [resp. $\left.C^{m}\left(\mathbf{M} ; \mathbf{R}^{2}\right)\right]$ the space of (resp. $\mathbf{R}^{2}$-valued) $m$ times continuously differentiable functions on $\mathbf{M}$.

Definition 3.1: A gauge potential $\mathbf{A} \in C^{m}\left(\Omega ; \mathbf{R}^{2}\right)(m$ $=0,1, \ldots, \infty)$ is said to be in the set $\mathfrak{U}_{m}$ if there exist realvalued functions $\phi_{1} \in C^{m+1}\left(\mathbf{R} \times \mathbf{S}_{2}\right)$ and $\phi_{2} \in C^{m+1}\left(\mathbf{S}_{1} \times \mathbf{R}\right)$ such that

$$
\begin{aligned}
& A_{1}(x, y)=\partial_{x} \phi_{1}(x, y), \quad(x, y) \in \mathbf{R} \times \mathbf{S}_{2}, \\
& A_{2}(x, y)=\partial_{y} \phi_{2}(x, y), \quad(x, y) \in \mathbf{S}_{1} \times \mathbf{R},
\end{aligned}
$$

where $\partial_{x}$ and $\partial_{y}$ denote the usual partial differential operators in $x$ and $y$, respectively.

For an open set $\mathbf{M} \subset \mathbf{R}^{2}$, we denote by $C_{0}^{m}(\mathbf{M})$ the space of functions in $C^{m}(\mathbf{M})$ with compact support in $\mathbf{M}$. Let

$$
\begin{aligned}
& \mathscr{D}_{1}^{m}=C_{0}^{m}\left(\mathbf{R} \times \mathbf{S}_{2}\right), \\
& \mathscr{D}_{2}^{m}=C_{0}^{m}\left(\mathbf{S}_{1} \times \mathbf{R}\right) .
\end{aligned}
$$

For all $m \geqslant 1$ and $A \in \mathfrak{U}_{0}$, we have

$$
\mathscr{D}_{j}^{m} \subset D\left(p_{j}\right) \cap D\left(A_{j}\right), \quad j=1,2 .
$$

Theorem 3.2: Let $m \geqslant 1$ and $\mathbf{A} \in \mathfrak{U}_{m-1}$. Then each $P_{j}$ is essentially self-adjoint on $\mathscr{D}_{j}^{m}$.
Proof: Under the assumption, $\phi_{1} \in C^{m}\left(\mathbf{R} \times \mathbf{S}_{2}\right)$ and $\phi_{2} \in C^{m}\left(\mathbf{S}_{1} \times \mathbf{R}\right)$. Hence, for each $j=1,2, e^{i q \phi_{j}}$ is a unitary operator such that $e^{i q \phi_{j}} \mathscr{D}_{j}^{m}=\mathscr{D}_{j}^{m}$. We have

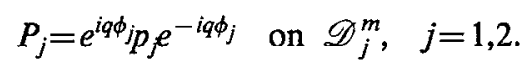

Hence we need only to show that $p_{j}$ is essentially selfadjoint on $\mathscr{D}_{j}^{m}$. In the standard identification $L^{2}\left(\mathbf{R}^{2}\right)$ $=L^{2}(\mathbf{R}) \otimes L^{2}(\mathbf{R})$, we can identify $p_{1}$ with $-i D_{x} \otimes I(I$ denotes identity). It is well known that $-i D_{x}$ is essentially self-adjoint on $C_{0}^{\infty}(\mathbf{R})$ and hence on $C_{0}^{m}(\mathbf{R})$ for all $m \geqslant 1$. Note that

$$
C_{0}^{m}(\mathbf{R}) \hat{\otimes} C_{0}^{m}\left(\mathbf{S}_{2}\right) \subset \mathscr{D}_{1}^{m},
$$

where $\hat{\otimes}$ denotes algebraic tensor product. Hence it follows that $p_{1}$ is essentially self-adjoint on $\mathscr{D}_{1}^{m}$. Similarly we can prove the essential self-adjointness of $p_{2}$ on $\mathscr{D}_{2}^{m}$.

Theorem 3.2 shows that for all $m \geqslant 1$, every $\mathbf{A} \in \mathfrak{l}_{m-1}$ satisfies the assumption of Theorem 2.1.

Example 3.3: Let $\lambda_{n} \in \mathbf{R}, n=1, \ldots, N$, be constants and $l_{n}, k_{n}$, be non-negative integers satisfying $2 k_{n} \geqslant l_{n}+1$. Let

$$
\begin{aligned}
& A_{1}(x, y)=\sum_{n=1}^{N} \frac{\lambda_{n}\left(x-a_{n 1}\right)^{l_{n}}\left(y-a_{n 2}\right)^{2 k_{n}-l_{n}-1}}{\left|\mathbf{r}-\mathbf{a}_{n}\right|^{2 k_{n}}}, \\
& A_{2}(x, y)=\sum_{n=1}^{N} \frac{\lambda_{n}\left(y-a_{n 2}\right)^{l_{n}}\left(x-a_{n 1}\right)^{2 k_{n}-l_{n}-1}}{\left|\mathbf{r}-\mathbf{a}_{n}\right|^{2 k_{n}}} .
\end{aligned}
$$

Then $A_{j}$ is in $C^{\infty}(\Omega)$. Let

$$
F_{n}(t)=\int_{0}^{t} \frac{\lambda_{n} s^{l_{n}}}{\left(1+s^{2}\right)^{k_{n}}} d s+C
$$

with a constant $C \in \mathbf{R}$ and

$$
\begin{aligned}
& \phi_{1}(x, y)=\sum_{n=1}^{N} F_{n}\left(\frac{x-a_{n 1}}{y-a_{n 2}}\right), \\
& \phi_{2}(x, y)=\sum_{n=1}^{N} F_{n}\left(\frac{y-a_{n 2}}{x-a_{n 1}}\right) .
\end{aligned}
$$

Then $\phi_{1} \in C^{\infty}\left(\mathbf{R} \times \mathbf{S}_{2}\right), \phi_{2} \in C^{\infty}\left(\mathbf{S}_{1} \times \mathbf{R}\right)$, and (3.1) and (3.2) hold. Hence $\mathbf{A}=\left(A_{1}, A_{2}\right) \in \mathfrak{U}_{\infty}$.

Example 3.4: Let $k$ be a non-negative integer, $\lambda_{\alpha \beta}^{(n)} \in \mathbf{R}$ $(n=1, \ldots, N, \alpha, \beta=1, \ldots, k)$ be constants, and

$$
\begin{aligned}
& A_{1}(x, y)=-\sum_{n=1}^{N} \sum_{\alpha, \beta=0}^{k} \lambda_{\alpha \beta}^{(n)} D_{x}^{\alpha} D_{y}^{\beta}\left(\frac{y-a_{n 2}}{\left|\mathbf{r}-\mathbf{a}_{n}\right|^{2}}\right), \\
& A_{2}(x, y)=\sum_{n=1}^{N} \sum_{\alpha, \beta=0}^{k} \lambda_{\alpha \beta}^{(n)} D_{x}^{\alpha} D_{y}^{\beta}\left(\frac{x-a_{n 1}}{\left|\mathbf{r}-\mathbf{a}_{n}\right|^{2}}\right) .
\end{aligned}
$$


It is obvious that $A_{f} \in C^{\infty}(\Omega)$. This example is a generalization of the one given in Ref. 1, which is the case of $N=1$ and $k=0$ with $\mathbf{a}_{1}=0$.

Let

$$
\begin{gathered}
\phi_{1}(x, y)=-\sum_{n=1}^{N} \sum_{\alpha, \beta=0}^{k} \lambda_{\alpha \beta}^{(n)} \partial_{x}^{\alpha} \partial_{y}^{\beta} \operatorname{Arctan}\left(\frac{x-a_{n 1}}{y-a_{n 2}}\right), \\
\phi_{2}(x, y)=\sum_{n=1}^{N} \sum_{\alpha, \beta=0}^{k} \lambda_{\alpha \beta}^{(n)} \partial_{x}^{\alpha} \partial_{y}^{\beta} \operatorname{Arctan}\left(\frac{y-a_{n 2}}{x-a_{n 1}}\right), \\
(x, y) \in \Omega .
\end{gathered}
$$

Then $\phi_{1} \in C^{\infty}\left(\mathbf{R} \times \mathbf{S}_{2}\right), \phi_{2} \in C^{\infty}\left(\mathbf{S}_{1} \times \mathbf{R}\right)$, and (3.1) and (3.2) hold. Hence $\mathbf{A}=\left(A_{1}, A_{2}\right) \in \mathfrak{U}_{\infty}$.

Noting that

$$
\begin{aligned}
& \frac{y-a_{n 2}}{\left|\mathbf{r}-\mathbf{a}_{n}\right|^{2}}=D_{y} \log \left|\mathbf{r}-\mathbf{a}_{n}\right|, \\
& \frac{x-a_{n 1}}{\left|\mathbf{r}-\mathbf{a}_{n}\right|^{2}}=D_{x} \log \left|\mathbf{r}-\mathbf{a}_{n}\right|,
\end{aligned}
$$

and using the fact that

$$
\left(D_{x}^{2}+D_{y}^{2}\right) \log \left|\mathbf{r}-\mathbf{a}_{n}\right|=2 \pi \delta\left(\mathbf{r}-\mathbf{a}_{n}\right),
$$

we obtain

$$
B(\mathbf{r})=2 \pi \sum_{n=1}^{N} \sum_{\alpha, \beta=0}^{k} \lambda_{\alpha \beta}^{(n)} D_{x}^{\alpha} D_{y}^{\beta} \delta\left(\mathbf{r}-\mathbf{a}_{n}\right) .
$$

Hence, the magnetic field $B$ is concentrated on the points $\mathrm{a}_{n}, n=1, \ldots, N$. In particular, (2.2) is satisfied. We can show that

$$
\gamma_{n}(\mathbf{A})=2 \pi \lambda_{00}^{(n)} .
$$

Hence it follows from Lemma 2.4 that

$$
\Phi_{s, t}^{\mathbf{A}}(x, y)=2 \pi \epsilon(s) \epsilon(t) \sum_{\mathbf{z}_{n} \in D(x, y, s, t)} \lambda_{00}^{(n)}
$$

This formula shows that the magnetic flux associated with the vector potential $\mathbf{A}=\left(A_{1}, A_{2}\right)$ given by (3.3) and (3.4) is locally quantized if and only if for all $n=1, \ldots, N$, $q \lambda_{00}^{(n)}$ is an integer. Therefore, in the present example, Theorem 2.3 implies the following: $\bar{P}_{1}$ and $\bar{P}_{2}$ strongly commute if and only if each $q \lambda_{o O}^{(n)}(n=1, \ldots, N)$ is an integer.

\section{REPRESENTATION OF THE CCRS}

In this section we discuss the relevance of the previous results to representation of the CCRs. We first note a simple fact.
Lemma 4.1: Let $X_{j}, j=1,2$, be the multiplication operators by the coordinate functions $x$ and $y$, respectively, and set

$$
\mathscr{D}=C_{0}^{2}(\Omega)
$$

Suppose that $\mathbf{A} \in C^{\mathrm{l}}\left(\Omega ; \mathbf{R}^{2}\right)$. Then $\left\{X_{j} P_{j} \mid j=1,2,\right\}$ satisfy the CCRs on $\mathscr{D}$ :

$$
\begin{aligned}
& {\left[X_{j}, X_{k}\right] \Psi=\left[P_{j}, P_{k}\right] \Psi=0,} \\
& {\left[X_{j}, P_{k}\right] \Psi=i \delta_{j k} \Psi, \quad \Psi \in \mathscr{D}, j, k=1,2,}
\end{aligned}
$$

if and only if (2.2) holds.

As for the CCRs in the Weyl form, we have the following result.

Theorem 4.2: Under the assumption of Theorem 2.1, the set $\left\{X_{j}, \bar{P}_{j} \mid j=1,2\right\}$ of self-adjoint operators fulfils the Weyl relations if and only if the magnetic flux associated with $\mathbf{A}$ is locally quantized.

Proof: In the same way as in the proof of Theorem 2.1, we can prove the following formula:

$$
e^{i S X_{j}} e^{i t \bar{P}_{k}}=e^{-i s t \delta} \delta_{j k} e^{i t \vec{P}_{k}} e^{i s X_{j}}, \quad j, k=1,2, \quad s, t \in \mathbf{R} .
$$

Obviously $X_{1}$ and $X_{2}$ strongly commute. Combining these facts with Theorem 2.3, we obtain the desired result.

Theorem 4.2 can be rephrased as follows: Under the assumption of Theorem $2.1,\left\{X_{j}, \bar{P}_{j} \mid j=1,2\right\}$ is equivalent to the Schrödinger representation of the CCRs if and only if the magnetic flux associated with $\mathbf{A}$ is locally quantized. This may be regarded as a representationtheoretical characterization for the local quantization of magnetic flux. Theorem 4.2, together with Lemma 4.1, also implies that, if (2.2) holds for A satisfying the assumptions of Theorem 2.1 and Lemma 4.1, but, the magnetic flux is not quantized locally, then $\left\{X_{j}, \bar{P}_{j} \mid j=1,2\right\}$ is a representation of the CCRs not equivalent to the Schrödinger representation. In Example 3.4, this situation occurs if some $q \lambda_{00}^{(n)}$ is not an integer. In the case of $N=1$, $k=0$, with $\mathbf{a}_{1}=0$, this fact has been pointed out in Ref. 1 .

\footnotetext{
${ }^{1}$ H. Reeh, J. Math. Phys. 29, 1535 (1988).

${ }^{2}$ E. Nelson, Ann. Math. 70, 572 (1959); see also Sec. VIII. 5 in Ref. 5. ${ }^{3}$ K. Schmüdgen, J. Funct. Anal. 50, 8 (1983).

${ }^{4}$ K. Schmüdgen, Publ. RIMS, Kyoto Univ. 19, 601 (1983).

${ }^{5} \mathrm{M}$. Reed and B. Simon, Methods of Modern Mathematical Physics I (Academic, New York, 1972).

${ }^{6}$ M. Reed and B. Simon, Methods of Modern Mathematical Physics II (Academic, New York, 1975).

${ }^{7}$ I. M. Gel'fand and G. E. Shilov, Generalized Functions, Vol. 2 (Academic, New York, 1968).
} 
Journal of Mathematical Physics is copyrighted by the American Institute of Physics (AIP). Redistribution of journal material is subject to the AIP online journal license and/or AIP copyright. For more information, see http://ojps.aip.org/jmp/jmpcr.jsp Copyright of Journal of Mathematical Physics is the property of American Institute of Physics and its content may not be copied or emailed to multiple sites or posted to a listserv without the copyright holder's express written permission. However, users may print, download, or email articles for individual use. 